\title{
MODELING THE LIGHT CURVE OF THE TRANSIENT SCP06F6
}

\author{
Emmanouil Chatzopoulos ${ }^{1}$, J. Craig Wheeler ${ }^{1}$, and J. Vinko ${ }^{1,2}$

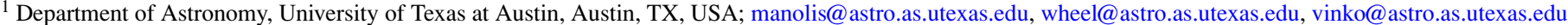 \\ 2 Department of Optics and Quantum Electronics, University of Szeged, Hungary \\ Received 2009 January 28; accepted 2009 September 10; published 2009 September 30
}

\begin{abstract}
We consider simple models based on core collapse or pair-formation supernovae ( $\mathrm{SNe}$ ) to account for the light curve of the transient SCP06F6. A radioactive decay diffusion model provides estimates of the mass of the required radioactive nickel and the ejecta as functions of the unknown redshift. An opacity change such as by dust formation or a recombination front may account for the rapid decline from maximum. Within this class of model, the redshift must be less than $z \sim 1$ or the nickel mass would exceed the total mass of the ejecta; the radiated energy would exceed the kinetic energy, and kinematic and photometric estimates of the radius would disagree. We particularly investigate two specific redshifts: $z=0.143$, for which Gaensicke et al. have proposed that the unidentified broad absorption features in the spectrum of SCP06F6 are $\mathrm{C}_{2}$ Swan bands, and $z=0.57$ based on a crude agreement with the $\mathrm{Ca} \mathrm{H} \& \mathrm{~K}$ and UV iron-peak absorption features that are characteristic of $\mathrm{SNe}$ of various types. For the lower redshift, we obtain a nickel mass of $0.3 M_{\odot}$ and an ejected envelope mass of $\sim 38 M_{\odot}$, while for the latter case we find $4.8 M_{\odot}$ and $20 M_{\odot}$, respectively, for fiducial parameters. The kinetic energy of the ejecta, while dependent on uncertain parameters, is generally large, $\sim 10^{52} \mathrm{erg}$, throughout this range of redshift. The ejected masses and kinetic energies are smaller for a more tightly constrained model invoking envelope recombination. We also discuss the possibilities of circumstellar matter (CSM) shell diffusion and shock interaction models. In general, optically thick CSM diffusion models can fit the data with the underlying energy coming from an energetic buried SN. Models in which the CSM is of lower density so that the shock energy is both rapidly thermalized and radiated tend not to be self-consistent. We suggest that a model of SCP06F6 worth further exploration is one in which the redshift is $\sim 0.57$, the spectral features are $\mathrm{Ca}$ and iron-peak elements, and the light curve is powered by the diffusive release of a substantial amount of energy from nickel decay or from an energetic SN buried in the ejecta of an LBV-like event.
\end{abstract}

Key words: circumstellar matter - hydrodynamics - stars: evolution - supernovae: general - supernovae: individual (SNSCP06F6)

Online-only material: color figures

\section{INTRODUCTION}

Recently discovered very luminous supernovae (SNe), such as SN 2006gy (Quimby 2006; Smith et al. 2007), SN 2005ap (Quimby et al. 2007a), SN 2006tf (Quimby et al. 2007b, 2007c; Smith et al. 2008) and SN 2008es (Yuan et al. 2008; Gezari et al. 2009; Miller et al. 2009) introduce evidence for new SN phenomena. The discovery of SN 2006gy triggered a rich discussion of the nature of this event, and a number of models were proposed to interpret the observed light curve (Ofek et al. 2007; Smith et al. 2007; Smith \& McCray 2007; Woosley et al. 2007; Agnoletto et al. 2008). SN 2006gy is classified as a Type IIn event that peaked at $V \sim 2270$ days after the explosion and radiated away more than $10^{51} \mathrm{erg}$ of energy. Radioactive ${ }^{56}$ Co decay fits of the light curve of SN 2006gy imply extraordinary amounts of initial nickel mass of the order of $22 M_{\odot}$ (Smith et al. 2007). The detection of soft unabsorbed X-rays by $X M M$-Newton indicated that the extended circumstellar matter (CSM) environment of SN 2006gy is of low density. Agnoletto et al. (2008) challenged this hypothesis by considering highly opaque clumps distributed around SN 2006gy from which they derive a nickel mass estimate of about $3 M_{\odot}$ (see Smith et al. 2009 for an extensive summary). SN 2006gy might be consistent with a model of a pair-formation SN (Rakavy \& Shaviv 1967; Barkat et al. 1967; Smith et al. 2007), but SN 2005ap, which is even brighter but with a narrower light curve, cannot be (Quimby et al. 2007a).

Recently, another apparently ultraluminous transient event was presented by the Supernovae Cosmology Project, SCP06F6
(Barbary et al. 2008). The possible high brightness, slow rise to maximum (100 days), and strange spectral features of SCP06F6 are still under debate. There is no detected host galaxy consistent with the position of SCP06F6 although examples of low-mass star-forming galaxies have been found that might be consistent with the upper limits (Dolphin et al. 2001). Thus SCP06F6 could define a new class if it is actually associated with an SN explosion. Due to the peculiar spectral appearance of SCP06F6 and the lack of an identified host galaxy, its redshift remains uncertain.

We discuss the uncertainties in the redshift, spectrum, and luminosity in Section 2. In the present work, we examine a range of possibilities for the redshift and two special cases. We present a basic radioactive decay diffusion model plus the possible effects of a recombination front and dust formation in Section 3 and use it to place constraints on the distance, ejected mass, nickel mass, and kinetic energy in Section 4. In Section 5, we present models based on interaction of an SN shock with CSM. A discussion is presented in Section 6.

\section{UNCERTAIN REDSHIFT, SPECTRUM, AND LUMINOSITY}

Barbary et al. (2008) discussed the possibility of the extragalactic origin of SCP06F6 since it has a small projected distance from the center of the galaxy cluster CL $1432.5+3332.8$ which has a redshift of 1.112. They noted that at this redshift, the absorption feature at $5890 \AA$ aligns with $\mathrm{Mg}_{\text {II }} \lambda \lambda 2796,2803$, 


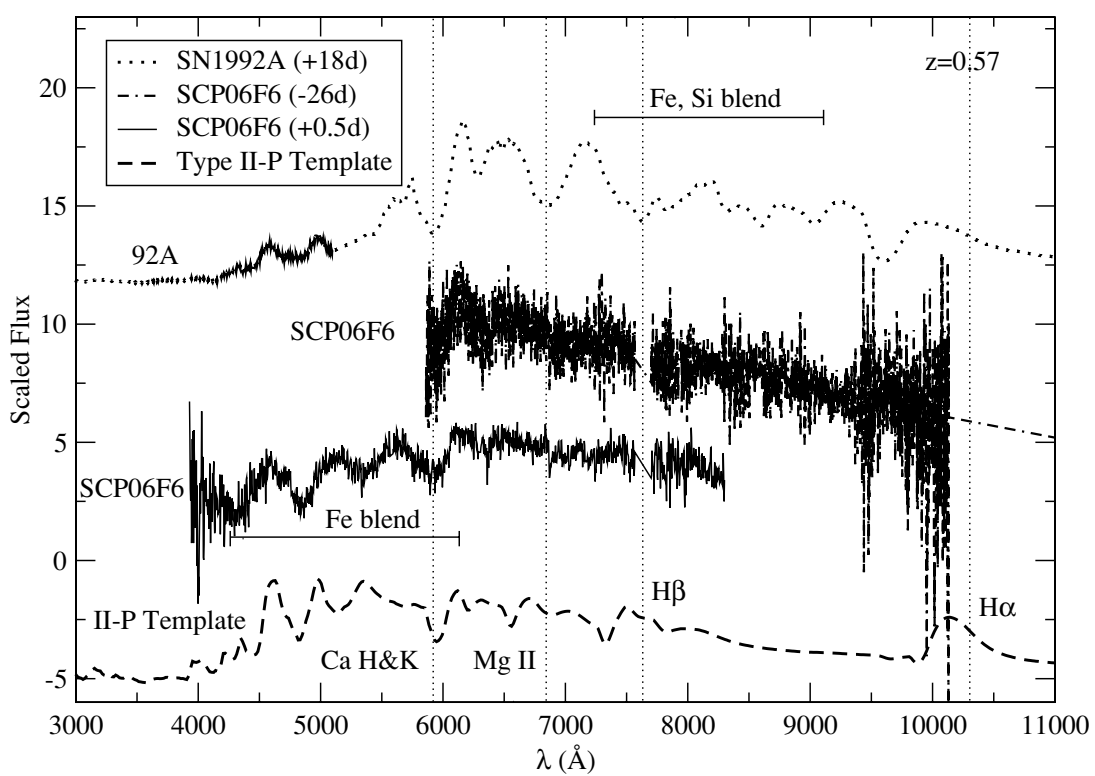

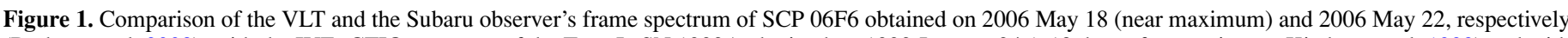

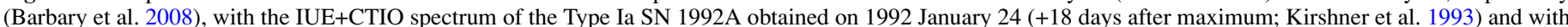

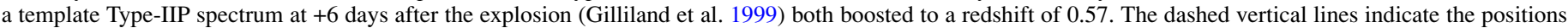

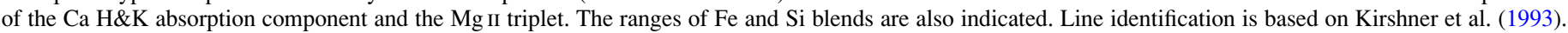

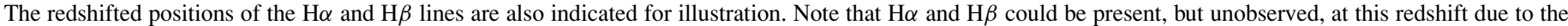
large redshift and telluric contamination.

but were unable to identify the other features. In particular, there are no features readily identifiable with hydrogen. In this work, we argue that the redshift cannot be above $z \sim 1$ through various constraints on the ejecta mass, luminosity, and radius.

One special case corresponds to $z=0.143$, as proposed by Gaensicke et al. (2008) by considering SCP06F6 as the explosion of a massive carbon-rich star with a cool and optically thick atmosphere. Gaensicke et al. (2008) propose that the broad "absorption" features present in the blue end of the spectrum of SCP06F6 (Figure 1) are molecular $\mathrm{C}_{2}$ bands (the Swan bands) originating in the cool, optically thick atmosphere of a carbonrich progenitor. The presence of carbon molecules constrains the temperature of the atmosphere of the progenitor to be cool so that they are not destroyed. Gaensicke et al. (2008) find that carbon star spectra redshifted by 0.143 provide a decent fit to the combined VLT+Keck spectrum of SCP06F6 (see also Soker et al. 2008). Other features present in carbon star spectra, however, such as CN ( 77900 rest frame; Downes et al. 2004), do not seem to appear in the smoothed co-added spectra of SCP06F6 (VLT+Subaru+Keck). Other CN bands would fall too far to the blue to be constrained by the available spectral data. We return to this issue in Section 6.

We also investigate the case for $z \sim 0.57$ where we can crudely identify three of the four broad absorption features by redshifting the spectrum of the local Type Ia SN 1992A or a template representing a Type IIP (Gilliland et al. 1999) as seen in Figure 1. A redshift of $z \sim 0.57$ aligns the $\mathrm{Ca}$ II $\mathrm{H} \& \mathrm{~K}$ absorption with the minimum at $\sim 5900 \AA$ in SCP06F6. The two bluest minima crudely correspond to minima in the UV spectra of SN 1992A and the SN IIP template caused by overlapping lines of iron-peak elements (Harkness \& Wheeler 1987; Kirshner et al. 1993). Similar features are seen in other $\mathrm{SNe}$ of various spectral types with UV spectra (Bufano et al. 2009). The agreement is not good with the minimum at about $5300 \AA$ in SCP06F6 where the $\mathrm{SN}$ II template has a peak. Note that at this redshift, the continuum slopes redward of $4000 \AA$ (but not the spectral features) also roughly agree. The comparison in Figure 1 does not suggest that SCP06F6 is either a Type Ia or a Type II, but that SCP06F6 might have Ca II and iron-peak absorption in the rest-frame UV moving with velocities typical of SNe. For reference, we also indicate in Figure 1 where $\mathrm{H} \alpha$ and $\mathrm{H} \beta$ would fall for this redshift. $\mathrm{H} \alpha$ would fall off the red end of the spectra, and $\mathrm{H} \beta$ would fall near the gap in the spectra that is contaminated by telluric lines. For this redshift, the lack of evidence for $\mathrm{H}$ in the spectrum cannot be taken as firm evidence for lack of hydrogen in the ejecta. The same is not true for a redshift of 0.143 where hydrogen might be present in emission or absorption for a variety of models, including those not based on $\mathrm{SNe}$.

To approximate the bolometric light curve of SCP06F6, we use the Very Large Telescope (VLT) spectrum at maximum (Barbary et al. 2008) scaled to the $i$ - and $z$-band fluxes observed at the same epoch. We then approximate the spectral energy distribution (SED) of the object with a triangle peaked at $6000 \AA$ that extends from 3000 to $12000 \AA$. We calculate the integral to get the quasi-bolometric flux at maximum light, $F_{\text {bol }}$. This approximate method gives $F_{\text {bol, } \max }=3.09 \times 10^{-14} \mathrm{erg} \mathrm{s}^{-1}$ $\mathrm{cm}^{-2}$ which is in good agreement with the estimated value of $2.5 \times 10^{-14} \mathrm{erg} \mathrm{s}^{-1} \mathrm{~cm}^{-2}$ given in Barbary et al. (2008). We then scale the flux to the other epochs using the photometry in Table 1 of Barbary et al. (2008) assuming that the shape of the SED is roughly constant. We use the following formula to obtain bolometric luminosities:

$$
L_{\mathrm{bol}}=F_{\mathrm{bol}} 4 \pi r^{2}
$$

where $r$ is the luminosity distance of the object which is equal to $r=(1+z / 2) \times c z / H_{0}$, where $c$ is the speed of light, $z$ is the redshift of the object, and $H_{0}=70 \mathrm{~km} \mathrm{~s}^{-1} \mathrm{Mpc}^{-1}$ is the Hubble constant. This expression for $r$ corresponds to an empty universe and is a good approximation to the full $\Lambda \mathrm{CDM}$ expression. 


\section{A SIMPLE DIFFUSION MODEL FOR SCP06F6}

\subsection{Radioactive Diffusion Model}

We adopt the Arnett (1982) radioactive decay diffusion model as generalized by Valenti et al. (2008; see also Soderberg et al. 2008) to allow for both nickel and subsequent cobalt decay. We also allow for gamma-ray leakage. This simple model assumes a diffusive medium with a uniform density profile and a photosphere that expands linearly in time. In general, the solutions we find for SCP06F6 imply large nickel and ejecta masses. With these assumptions, we adopt the following expression for the diffusive luminosity released as a result of radioactive decay:

$$
\begin{aligned}
L(t)= & M_{\mathrm{Ni}} e^{-x^{2}}\left[\left(\epsilon_{\mathrm{Ni}}-\epsilon_{\mathrm{Co}}\right) \int_{0}^{x} 2 z e^{z^{2}-2 z y} d z+\epsilon_{\mathrm{Co}}\right. \\
& \left.\times \int_{0}^{x} 2 z e^{z^{2}-2 y z+2 z s} d z\right]\left(1-e^{-A t^{-2}}\right),
\end{aligned}
$$

where $x=t / t_{m}, t_{m}$ is the rise time to maximum (Arnett 2008), $y=t_{m} / 2 t_{\mathrm{Ni}}$ with $t_{\mathrm{Ni}}=8.8$ days, $s=t_{m}\left(t_{\mathrm{Co}}-t_{\mathrm{Ni}}\right) / 2 t_{\mathrm{Co}} t_{\mathrm{Ni}}$ with $t_{\mathrm{Co}}=111.3$ days, $M_{\mathrm{Ni}}$ is the initial nickel mass, $\epsilon_{\mathrm{Ni}}=3.9 \times 10^{10}$ $\mathrm{erg} \mathrm{s}^{-1} \mathrm{~g}^{-1}$, and $\epsilon_{\mathrm{Co}}=6.8 \times 10^{9} \mathrm{erg} \mathrm{s}^{-1} \mathrm{~g}^{-1}$ are the energy generation rates due to $\mathrm{Ni}$ and $\mathrm{Co}$ decay. The factor $\left(1-e^{-A t^{-2}}\right)$ accounts for the gamma-ray leakage, where large $A$ means that practically all gamma rays are trapped. The gamma-ray optical depth of the ejecta is taken to be $\tau_{\gamma}=\kappa_{\gamma} \rho R=A t^{-2}$, assuming spherical uniform density ejecta with radius $R=v t$ and the $\mathrm{Ni} / \mathrm{Co}$ confined in the center. This yields $A=\left(3 \kappa_{\gamma} M_{e j}\right) /\left(4 \pi v^{2}\right)$ which is controlled by the gamma-ray opacity $\kappa_{\gamma}$. The $t^{-2}$ scaling follows from homologous expansion which is one of the basic assumptions of the simple analytic models that we adopt here. In general, we find the effect of gamma-ray leakage to be small for the large masses implied in our models.

Thus we have three main fitting parameters that determine the nature of the model light curve: the initial ${ }^{56} \mathrm{Ni}$ mass that determines its peak, the rise time to maximum $t_{m}$ that determines its width, and $A$ that determines the amount of gamma-ray trapping and corresponds to a gamma-ray opacity. It is characteristic that, within this class of model, the nickel mass comes in only as a scaling parameter for the amplitude of the luminosity output. The more distant the source is, the brighter it is, since its intrinsic (rest frame) luminosity is higher compared to the observed luminosity. We thus expect a higher initial ${ }^{56} \mathrm{Ni}$ mass at higher redshifts.

Unlike the maximum brightness, the width of the light curve in the object rest frame decreases with respect to its observed value, since the rest-frame rise time, $t_{m, \text { rf }}$, decreases with redshift:

$$
t_{m}=\frac{t_{m, \mathrm{ob}}}{1+z}
$$

where $t_{m, \mathrm{ob}}$ is the observed rise time. Assuming that the photosphere expands with approximately constant speed, $v_{\mathrm{ph}}$, we adopt the ejecta mass as a function of the observed rise time given by Valenti et al. (2008):

$$
M_{\mathrm{ej}}(z)=\frac{3}{10} \frac{\beta c}{\kappa} v_{\mathrm{ph}} \frac{t_{m, \mathrm{ob}}^{2}}{(1+z)^{2}},
$$

where $\beta$ is an integration constant equal to about 13.8 (Arnett 1982; Valenti et al. 2008), $\kappa$ is the mean opacity, and we have used $v_{\mathrm{ph}}=\left(10 / 3 E_{\mathrm{KE}} / M_{\mathrm{ej}}\right)^{1 / 2}$, as appropriate for the outer edge of a homologously expanding sphere of constant density with ejecta mass $M_{\mathrm{ej}}$ and kinetic energy $E_{\mathrm{KE}}$. The ejecta mass is set by the rise time to maximum that depends on the redshift of the object. Thus we obtain a scaling of the ejecta mass with redshift within the context of this model for SCP06F6. The photospheric velocity of SN ejecta is estimated from the width of lines in their spectra. For Type II SNe, it typically varies within the range $4000-6000 \mathrm{~km} \mathrm{~s}^{-1}$, and for Type Ia SNe it is typically $10,000-15,000 \mathrm{~km} \mathrm{~s}^{-1}$. We note that the features of SCP06F6 are generally broader than those of the Type IIP template of Figure 1 and perhaps more represented by the features of the Type Ia. Barbary et al. (2008) gave $v_{\text {ph }} \simeq 12,000 \mathrm{~km} \mathrm{~s}^{-1}$. We adopt $v_{\mathrm{ph}}=10,000 \mathrm{~km} \mathrm{~s}^{-1}$ as a fiducial value and assume this to refer to the photospheric velocity in the rest frame of the SN for the models presented in Section 4. With assumed values of opacity we attempt to fit the observed light curve and to obtain constraints on physical properties of SCP06F6 that will in turn scale as a function of the redshift.

\subsection{The Effects of Recombination}

The light curve presented by Barbary et al. (2008) is roughly symmetric about the peak. The fact that SCP06F6 shows a symmetric light curve is not in contradiction with observed SNe characteristics. There are cases such as the Type Ib SN SN2005bf (Tominaga et al. 2005) for which the second peak has a very symmetric light curve. As we will show below, our simple radioactive diffusion models have difficulty accounting for the low values of the points 100 days after peak and hence reproducing the symmetric light curve. There are several factors that might account for this discrepancy. The fundamental assumption of the model of Section 3.1 is that the ejecta are optically thick. That may not be true at the last observed epoch.

An expression for the optical depth at maximum light can be written as

$$
\tau_{\max } \sim \kappa \bar{\rho} R \sim \frac{3 \kappa M_{\mathrm{ej}}}{4 \pi R^{2}} .
$$

Using the expression for $\kappa M_{\mathrm{ej}}$ from Equation (4) and the kinematic radius $R_{k, \text { max }}=v_{\mathrm{ph}} t_{\max } /(1+z)$ gives $\tau_{\max } \simeq c / v_{\mathrm{ph}}$, independent of redshift. For a photospheric velocity of 10,000 $\mathrm{km} \mathrm{s}^{-1}$, the optical depth would be about 30 , thus justifying the assumption of large optical depth at maximum light. Using the blackbody radius gives a similar estimate of large optical depth, but one that varies with redshift.

As the ejecta expand, the optical depth decreases as $\tau \simeq t^{-2}$. For constant opacity the optical depth at epoch 11 of Barbary et al. (2008; Table 1) is thus $\tau_{11} \simeq \tau_{\max }\left(t_{\max } / t_{11}\right)^{2} \simeq 30 / 3 \simeq 10$ for $\tau_{\max } \simeq 30, t_{\max }=100$ days, and $t_{11}=170$ days. If the opacity dropped by a factor of several after maximum due to recombination in some element or elements, the light curve might decline more rapidly than our models.

To incorporate the effects of recombination, we use the model developed by Arnett \& Fu (1989) to fit the light curve of SN1987A. This model considers the effects of the recombination of hydrogen or helium. After peak luminosity, the recombination front recedes inward, and the optical opacity drops dramatically. Thus the thermal energy generated by the presumed shock diffuses out more quickly, and the decline is faster than it would be without recombination. The light curve that results from this model depends on the following input parameters: the initial radius of the progenitor $R_{0}$, the nickel mass yield $M_{\mathrm{Ni}}$, the mass of the ejecta $M_{\mathrm{ej}}$, the kinetic and the thermal energy $E_{\mathrm{kin}}$ and $E_{\text {th }}$, respectively, the ionization temperature $T_{\text {ion }}$ and the optical 

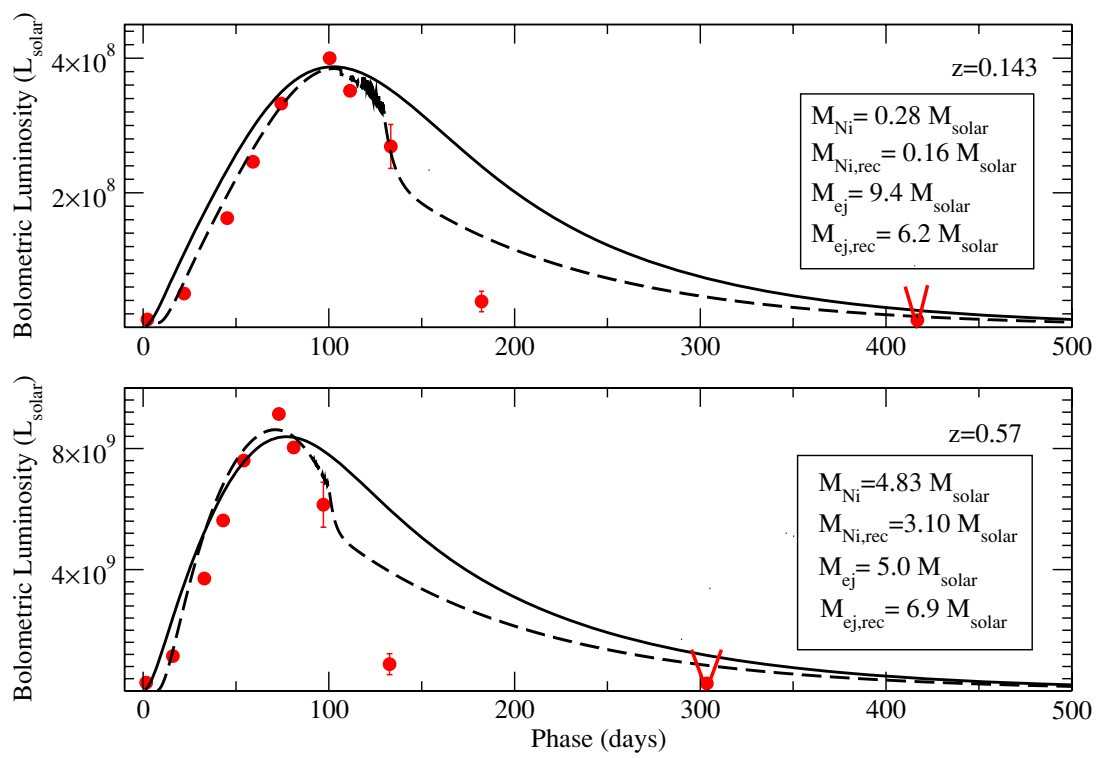

Figure 2. Rest-frame quasi-bolometric luminosity light curve of SCP 06F6 (Section 3.1) at redshifts of 0.143 (top panel) and 0.57 (bottom panel) (solid points). In each case, the solid line is a simple radioactive diffusion model (Section 3.1) and the dashed line is an illustration of the Arnett \& Fu (1989) model that includes the effects of recombination giving a decline in the optical opacity and thus a rapid post-maximum decline. The derived values of the original nickel mass and of the ejecta mass are given in the insets. Note that in this figure the basic model assumes $\kappa=0.1 \mathrm{~cm}^{2} \mathrm{~g}^{-1}$ and the corresponding recombination models assume $\kappa=0.4$ and $0.2 \mathrm{~cm}^{2} \mathrm{~g}^{-1}$, respectively, for $z=0.143$ and $z=0.57$ (see Tables 1 and 2).

(A color version of this figure is available in the online journal.)

Table 1

Summary of the Fiducial Physical Parameters of SCP06F6

\begin{tabular}{|c|c|c|c|c|c|c|}
\hline \multirow{2}{*}{$\begin{array}{c}\text { Parameter } \\
\kappa\left(\mathrm{cm}^{2} \mathrm{~g}^{-1}\right)\end{array}$} & \multicolumn{3}{|c|}{$z=0.143$} & \multicolumn{3}{|c|}{$z=0.57$} \\
\hline & 0.05 & 0.1 & 0.4 & 0.05 & 0.1 & 0.4 \\
\hline$t_{m, r f}$ (days) & 90 & 90 & 90 & 65 & 65 & 65 \\
\hline$M_{\mathrm{Ni}}\left(M_{\odot}\right)$ & 0.28 & 0.28 & 0.28 & 4.83 & 4.83 & 4.83 \\
\hline$\overline{M_{e j}\left(M_{\odot}\right)}$ & 75.4 & 37.7 & 9.4 & 39.4 & 19.7 & 4.9 \\
\hline$E_{\mathrm{th}} / 10^{50}(\mathrm{erg})$ & 0.91 & 0.91 & 0.91 & 14.0 & 14.0 & 14.0 \\
\hline$E_{\text {kin }} / 10^{51}(\mathrm{erg})$ & 42.0 & 21.0 & 5.3 & 5.5 & 11.0 & 2.8 \\
\hline$R_{b b}\left(10^{15} \mathrm{~cm}\right)$ & 1.7 & 1.7 & 1.7 & 7.8 & 7.8 & 7.8 \\
\hline$R_{\text {kin }}\left(10^{15} \mathrm{~cm}\right)$ & 7.5 & 7.5 & 7.5 & 5.5 & 5.5 & 5.5 \\
\hline$\tau_{\text {thick }}{ }^{\mathrm{a}}$ & 30 & 30 & 30 & 30 & 30 & 30 \\
\hline$\tau_{\text {thin }}{ }^{\mathrm{a}}$ & 0.001 & 0.003 & 0.011 & 0.04 & 0.08 & 0.32 \\
\hline$\dot{M}_{\text {thick }}\left(M_{\odot} \mathrm{yr}^{-1}\right)^{\mathrm{a}}$ & 3.0 & 1.5 & 0.38 & 2.22 & 1.11 & 0.28 \\
\hline$\dot{M}_{\text {thin }}\left(M_{\odot} \mathrm{yr}^{-1}\right)^{\mathrm{a}}$ & $2 \times 10^{-4}$ & $2 \times 10^{-4}$ & $2 \times 10^{-4}$ & $3 \times 10^{-3}$ & $3 \times 10^{-3}$ & $3 \times 10^{-3}$ \\
\hline
\end{tabular}

Notes. $v_{\mathrm{ph}}=10,000 \mathrm{~km} \mathrm{~s}^{-1}$ for all cases.

a The values of $\tau_{\text {thick }}$ and $\dot{M}_{\text {thick }}$ are estimated based on a model of an optically thick shell, and the values of $\tau_{\text {thin }}$ and $\dot{M}_{\text {thin }}$ are estimated based on a model of moderate optical depth for which the luminosity is quickly radiated behind the shock. The given mass-loss rates assume a "wind" velocity of $100 \mathrm{~km} \mathrm{~s}^{-1}$.

opacity $\kappa$. The values that we used to obtain the fits shown in Figure 2 are summarized in Table 2 . Also given in Table 2 is the mean ejecta velocity, $v_{\text {mean }}=\sqrt{2 E_{\mathrm{kin}} / M_{\mathrm{ej}}}$ implied by the model fits. The amount of nickel needed to power the event in the recombination model is less than it is if the recombination effects are not taken into account, as expected. Specifically, $M_{\mathrm{Ni}, \text { rec }} / M_{\mathrm{Ni}} \simeq 0.6$ for both redshift models, where $M_{\mathrm{Ni} \text {,rec }}$ is the mass of nickel that is required to power the peak of the light curve if the recombination effects are taken into account.

As seen in Figure 2, a recombination model can produce a roughly symmetric light curve around the peak that subsequently follows the cobalt decay. In our model for SCP06F6, we consider the composition of the ejecta unknown and we adopt the Thompson scattering opacity values of $0.4 \mathrm{~cm}^{2} \mathrm{~g}^{-1}$ at $z=0.143$, (a typical value for an $\mathrm{H}$-rich envelope) and $0.2 \mathrm{~cm}^{2} \mathrm{~g}^{-1}$ at $z$ $=0.57$ (a typical value for an H-poor envelope). As noted in
Section 2, there is no evidence for hydrogen or helium in the spectrum of SCP06F6, but if it is at a redshift of 0.57 , then the $\mathrm{H} \alpha$ line would be redshifted out of the range of the spectral coverage by VLT and Subaru as shown in Barbary et al. (2008) and $\mathrm{H} \beta$ would be contaminated by telluric lines (Figure 1). We are not able to accurately reproduce the light curve of SCP06F6, even when the recombination effects are taken into account, especially due to the fact that we cannot fit epoch 11 of Barbary et al. (2008). It should be noted that the purpose of the recombination models that we consider is to illustrate the effects of this process on the light curve of an $\mathrm{SN}$, and to show that recombination does tend to make the light curve more symmetric around the peak. More detailed models accounting for the density, composition, and optical depth structure and appropriate radiative transfer are beyond the scope of this work. 


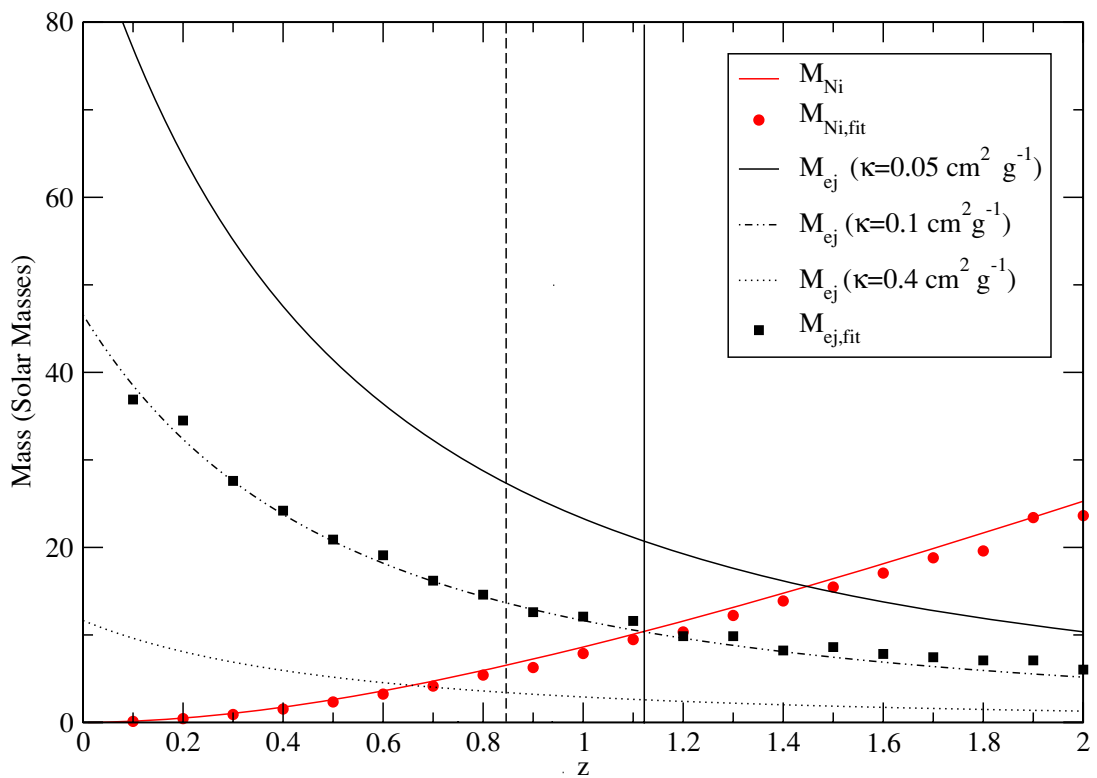

Figure 3. Dependence of the initial nickel mass (rising line) and the ejecta mass (decreasing lines) on redshift for SCP06F6. The line for the nickel mass is based on Equations (1) and (2). The lines for the ejecta mass are from Equation (4). The ejecta mass depends linearly with the photospheric velocity assumed to be 10,000 $\mathrm{km} \mathrm{s}^{-1}$ and inversely on the mean opacity, for which three choices are shown: $\kappa=0.05$ (solid), 0.1 (dotted dashed), and 0.4 (dotted) $\mathrm{cm}^{2} \mathrm{~g}^{-1}$. The filled circles and squares correspond to the best-fitting estimates of the nickel mass and the ejecta mass respectively calculated by the Monte Carlo $\chi^{2}$ minimization code (see the text). At redshifts to the right of the solid vertical line, the mass of radioactive nickel becomes larger than the total ejecta mass for $\kappa=0.1 \mathrm{~cm}^{2} \mathrm{~g}^{-1}$. This line thus defines the lower redshift boundary of a "forbidden region" in redshift space for this class of radioactive diffusion model. The dashed vertical line indicates the redshift at which the nickel mass is half the total ejected mass for $\kappa=0.1 \mathrm{~cm}^{2} \mathrm{~g}^{-1}$. The forbidden region extends to lower redshift for higher opacity and lower photospheric velocity. (A color version of this figure is available in the online journal.)

Table 2

Summary of the Parameters of the Recombination Models

\begin{tabular}{lcc}
\hline \hline \multicolumn{1}{c}{ Parameter } & $z=0.143$ & $z=0.57$ \\
\hline$R_{0}\left(10^{11} \mathrm{~cm}\right)$ & 2 & 2 \\
$M_{\mathrm{Ni}}\left(M_{\odot}\right)$ & 0.16 & 3.1 \\
$M_{e j}\left(M_{\odot}\right)$ & 6.2 & 6.9 \\
$E_{\text {th }}\left(10^{51} \mathrm{erg}\right)$ & 1.5 & 1.0 \\
$E_{\mathrm{kin}}\left(10^{51} \mathrm{erg}\right)$ & 1.5 & 3.0 \\
$T_{\text {ion }}(\mathrm{K})$ & 5500 & 10000 \\
$\kappa\left(\mathrm{cm}^{2} \mathrm{~g}^{-1}\right)$ & 0.4 & 0.2 \\
$v_{\text {mean }}\left(\mathrm{km} \mathrm{s}^{-1}\right)$ & 3900 & 2700
\end{tabular}

\subsection{The Effect of Dust}

There could also be dust formation at late epochs. At epoch 11 (Table 1, Barbary et al. 2008) the observed flux is $\sim 7$ times lower than the flux that is predicted by the model (insensitive to redshift). Thus we can estimate the optical depth due to a hypothetical dust absorption using the formula $F_{11}=F_{0,11} e^{-\tau_{\text {dust }}}$, where $F_{11}$ is the flux at epoch $11, F_{0,11}$ is the flux at epoch 11 as predicted by the radioactive decay diffusion model, and $\tau_{\text {dust }}$ is the dust optical depth. This yields $\tau_{\text {dust }} \simeq 1.9$ if we consider a spherical, optically thin dust shell at the outer edge of the SN ejecta with radius $\simeq R_{11}=v_{\text {ph }} t_{11}$ and thickness $d R \ll R_{11}$. The required dust mass is then $M_{\text {dust, } 11}=$ $4 \pi R_{11}^{2} \rho_{\text {dust }} d R$, and the dust optical depth $\tau_{\text {dust }}=\kappa_{\text {dust }} \rho_{\text {dust }} d R$. Combining these equations yields $M_{\text {dust }}=\tau /\left(\kappa_{\text {dust }} 4 \pi R_{11}^{2}\right)$, where $\kappa_{\text {dust }}$ is the opacity of the dust grains. We adopt the value $\kappa_{\text {dust }}=10^{4} \mathrm{~cm}^{2} \mathrm{~g}^{-1}$ as a gross estimate, using the Draine (2003) model extinction curves for Milky Way dust grain composition. For this choice of parameters we estimate the dust mass to be $\sim 3 \times 10^{-4} M_{\odot}$. This value is in agreement with values that are estimated for other Type-II SNe (Kotak et al. 2005, 2009).
If the dust formation took place well inside the ejecta, for example, at $v \simeq 1000 \mathrm{~km} \mathrm{~s}^{-1}$, as in Type II-P SNe (Kotak et al. 2009), then the dust mass would be 100 times higher than the value estimated above, on the order of $0.02 M_{\odot}$. This is somewhat higher than that has been observed in other $\mathrm{SNe}$, but still not unrealistic, taking into account that the dust masses derived in this way are only lower limits. If the dust distribution is not uniform and optically thin but rather it is distributed in optically thick clumps (Ercolano et al. 2007), then the total dust mass could be much higher than that derived from the optically thin model.

Note that the energy absorbed by dust is expected to be reradiated as mid-IR photons after the dust particles (or clumps) are heated up to $T_{\text {dust }} \sim 600-1000 \mathrm{~K}$. Thus, strictly speaking, the true bolometric luminosity remains unchanged in this case. In Section 2, we estimated the bolometric luminosity by assuming that all radiation comes from the optical and near-IR regimes. The Arnett model (Equation (2)) also uses essentially the same assumption due to the thermalization of the gamma rays. Within this context, the optical photons absorbed by dust can be considered as "lost," even though their energy is expected to re-appear as a mid-IR excess, outside the original SED.

\section{CONSTRAINTS ON SCP06F6}

\subsection{General Redshift Constraints}

Here we calculate the redshift dependence of the physical parameters of the Arnett model given in Equation (2), without recombination. Figure 3 shows how the ejecta mass and the nickel mass depend on redshift based on fits to the light curve for various choices of the mean opacity. The recombination models have the same redshift dependence, but the nickel masses are about $40 \%$ lower, the ejecta masses are about $30 \%$ lower, and the kinetic energies are lower by a factor of 2-3 compared to 


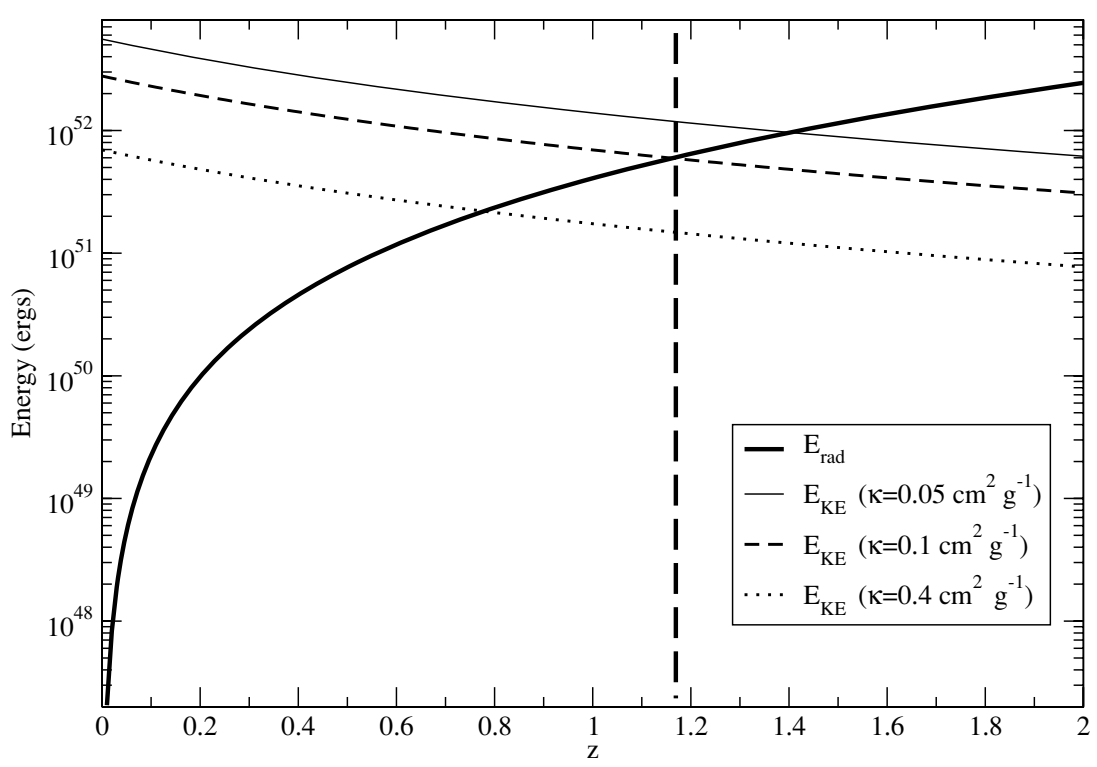

Figure 4. Dependence of the total radiated energy (thick solid line) and the kinetic energy of the ejecta on redshift for SCP06F6. Three choices of the mean opacity are shown for the kinetic energy (see Figure 1). The region at redshifts to the right of the dashed vertical line at $z=1.18$ is forbidden for $\kappa=0.1 \mathrm{~cm}^{2} \mathrm{~g}^{-1}$ since the radiated energy becomes larger than the kinetic energy of the ejecta. This forbidden region extends to lower redshifts for larger opacity.

our fiducial models with the same opacity. The ejecta mass for the basic radioactive diffusion models is calculated using Equation (4) adopting $t_{m, \mathrm{ob}}=100$ days for the rise time to maximum in the observer's frame and adopting $v_{\text {ph }}=10,000$ $\mathrm{km} \mathrm{s}^{-1}$ as the fiducial velocity. The nickel mass is calculated by assuming $t_{\max }=t_{m}$ and solving Equation (2) for $M_{\mathrm{Ni}}$ given the observed value of maximum luminosity, which scales with redshift according to Equation (2). We consider values of the mean opacity ranging from $0.05 \mathrm{~cm}^{2} \mathrm{~g}^{-1}$ as might be representative of a metal-rich composition (Sutherland \& Wheeler 1984; Soderberg et al. 2008) up to $0.40 \mathrm{~cm}^{2} \mathrm{~g}^{-1}$ as might be representative of electron scattering in a pure hydrogen plasma. Figure 3 also shows the best-fitting results for the nickel mass and the ejecta mass in the case of $\kappa=0.1 \mathrm{~cm}^{2} \mathrm{~g}^{-1}$ for the same redshift range and with redshift increments of 0.1 (filled circles and squares, respectively). To determine the best-fitting parameters $\left(M_{\mathrm{Ni}}, t_{m}\right.$, and $\left.A\right)$ for each redshift, we developed a simple Monte Carlo chi-square minimization code that scans through all the parameter space and finds the minimum $\chi^{2}$ value and the parameters that correspond to that value. As can be seen in Figure 3, the best-fitting results are in very good agreement with the analytic ones assuming $t_{m, \mathrm{ob}}=100$ days as a fixed parameter.

The region above redshift of $\sim 1.1$ is forbidden since the required nickel mass would exceed the required ejecta mass to account for the width of the light curve even for rather small opacity. The region to the right of the dashed vertical line at $z \sim 0.8$ (for $\kappa=0.1 \mathrm{~cm}^{-2} \mathrm{~g}^{-1}$ ) is also forbidden in practice because it is unlikely that the nickel mass exceeds half the total ejecta mass in an astrophysically realistic model of, for example, a pair-formation or core-collapse SN. These redshift limits get tighter for larger mean opacities or lower photospheric velocities. For electron scattering in a pure ionized hydrogen plasma, the redshift would have to be less than about 0.65 for this sort of model to be self-consistent. Invoking recombination does not change this constraint substantially since $M_{\mathrm{ej}} / M_{\mathrm{Ni}}$ is about the same (Tables 1 and 2). A nickel mass of $1 M_{\odot}$ that would be characteristic of a Type Ia is obtained at a redshift of about 0.3 . At this redshift, the ejecta would exceed $30 M_{\odot}$ for a mean opacity of $\kappa \lesssim 0.1 \mathrm{~cm}^{2} \mathrm{~g}^{-1}$, so the explosion is certainly not that of an exploding white dwarf.

Figure 4 shows the scaling with redshift of the kinetic energy, $E_{\mathrm{KE}}=1 / 2 M_{\mathrm{ej}} v_{\text {mean }}^{2}$, and the total radiated energy, $E_{\mathrm{rad}}$. The kinetic energy is determined from the ejecta mass, assuming a mean velocity of $v_{\text {mean }}=\sqrt{3 / 5} v_{\mathrm{ph}}=7800 \mathrm{~km} \mathrm{~s}^{-1}$ and the same range of mean opacity as before. Note that the kinetic energy is especially sensitive to the assumed photospheric velocity, scaling as $E_{\mathrm{KE}} \propto v_{\mathrm{ph}}^{3}$ (from Equation (4)). The more tightly constrained recombination models require a lower $E_{\text {kin }} / M_{\text {ej }}$ and hence $v_{\text {mean }}$ than our fiducial radioactive diffusion models (Table 2). This raises the possibility that our fiducial models presented here and in Figure 4 overestimate the ejecta velocity and kinetic energy. The radiated energy is obtained by integrating the bolometric luminosity over the whole light curve. Since a strong shock distributes energy equally between kinetic energy and thermal energy in its wake, and some thermal energy is likely to be subsequently converted to kinetic energy by adiabatic expansion and $P d V$ work, it is unlikely that the radiated energy can exceed the kinetic energy. This constraint gives another limit on the redshift, $z \lesssim 1.1$ for $\kappa=0.1 \mathrm{~cm}^{-2}$ $\mathrm{g}^{-1}$, as shown in Figure 3. Note that if the opacity were larger or the photospheric velocity smaller, this constraint would also get tighter.

An estimate of the radius of the photosphere can be obtained from kinematics $\left(R=v_{\mathrm{ph}} t\right)$ or emissivity (a blackbody radius). Figure 5 gives the radius estimated at maximum light in these two ways assuming a constant rest-frame photospheric velocity of $10,000 \mathrm{~km} \mathrm{~s}^{-1}$ and a constant rest-frame blackbody temperature of $5000 \mathrm{~K}$. Note that these two estimates only agree at a single redshift. Each estimate is uncertain, so we also denote the range over which the radii agree within a factor of 2 or a factor of 3 . The latter restricts the range of redshift to $z \sim 0.2-0.9$.

Another consistency check follows from noticing that the slope of the continuum varies little over the three epochs for which spectra were given by Barbary et al. (2008). We have estimated the effective temperature in our models at these epochs using the bolometric luminosity and the kinematic radius from 


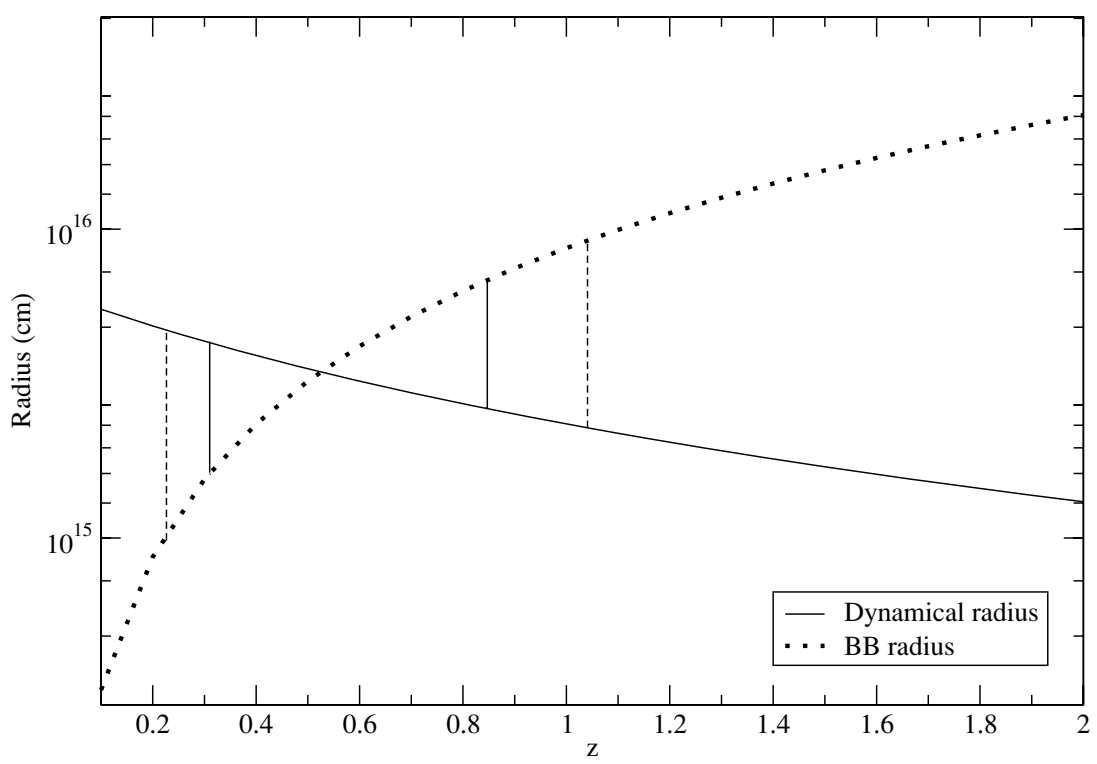

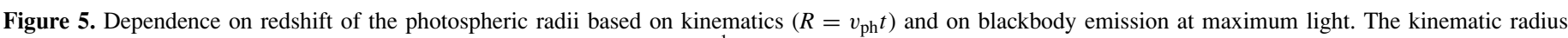

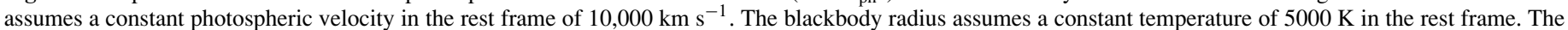
vertical lines indicate the redshift range over which these estimates of the radius agree within a factor of 2 (solid line) and a factor of 3 (dashed line), respectively.

Figure 5. The effective temperature derived in this way (that is formally $5000 \mathrm{~K}$ at $z \sim 0.5$ ) varies by $\lesssim 300 \mathrm{~K}$ over the spectral epochs, basically consistent with the observations.

\subsection{Special Case 1: $z=0.143$}

We next examine the special case of $z=0.143$ as suggested by Gaensicke et al. (2008), who fit the spectrum of SCP06F6 with redshifted carbon-rich stellar spectra from the Sloan Digital Sky Survey (SDSS) database. The result of the analysis for $z=0.143$ is shown in the top panel of Figure 2. The best fit gives a value of $M_{\mathrm{Ni}}=0.28 M_{\odot}$ and $M_{\mathrm{ej}}=37.74\left(\frac{\kappa}{0.1 \mathrm{~cm}^{-2} \mathrm{~g}^{-1}}\right)^{-1}$ $\left(\frac{v_{p h}}{10,000 \mathrm{~km} \mathrm{~s}^{-1}}\right) M_{\odot}$. The best-fitting $A$ parameter which controls the gamma-ray leakage implies a small gamma-ray opacity $\kappa_{\gamma}=5 \times 10^{-4} \mathrm{~cm}^{2} \mathrm{~g}^{-1}$, whereas a typical gamma-ray opacity might be $\sim 0.03 \mathrm{~cm}^{2} \mathrm{~g}^{-1}$ (Colgate et al. 1980). Gamma-ray leakage is not an important effect for the large masses derived. The initial nickel mass is within the range as expected for typical $\mathrm{SNe}$ while the ejecta mass is rather large, due to the fact that SCP06F6 shows a significantly slow rise to maximum and a slow decline. Note that the ejecta mass varies inversely with the assumed, and uncertain, opacity (Equation (4)). This mass could be less if the mean opacity were greater than the value, $\kappa=0.1 \mathrm{~cm}^{-2} \mathrm{~g}^{-1}$ assumed here for illustration. It would also be less for smaller $v_{\text {phot }}$.

The value of the ejecta mass implies a total kinetic energy of $E_{\mathrm{KE}}=2.4 \times 10^{52}\left(\frac{\kappa}{0.1 \mathrm{~cm}^{-2} \mathrm{~g}^{-1}}\right)^{-1}\left(\frac{v_{\mathrm{ph}}}{10,000 \mathrm{~km} \mathrm{~s}^{-1}}\right)^{3}$ erg while a lower limit for the radiated energy is $9.1 \times 10^{49} \mathrm{erg}$. While the estimated radiated energy is roughly in accord with "normal" $\mathrm{SNe}$, the estimated kinetic energy is very large. For the assumed opacity, the implication is that the initial radius was rather small compared to the radius of the photosphere at maximum light so that substantial initial thermal energy was lost to adiabatic expansion. For a larger opacity and a photospheric velocity more in the range typical of Type II SNe, the estimated kinetic and radiated energies would be more nearly equivalent.

The top panel of Figure 2 also shows the results of a model that incorporates the effects of recombination on the light curve (Section 3.2) for this redshift case. The recombination temperature was found to be $T=5500 \mathrm{~K}$ with $\kappa=0.4 \mathrm{~cm}^{2} \mathrm{~g}^{-1}$ which is appropriate for an H-rich atmosphere, although there is no evidence for the presence of $\mathrm{H}$ in the spectrum if this was the correct redshift. For this model, the nickel mass, the ejecta mass, and the kinetic energy are lower than for the basic model, as mentioned above. The kinetic energy, and hence implicitly the mean velocity, was varied to produce the dashed curve in the top panel of Figure 2. Given the uncertainties, no attempt was made to determine the "best fit" over the full parameter range. This model shows that recombination could help to produce a roughly symmetric light curve and that this physics, in principle, is relevant to SCP06F6, although it was not possible to fit the last measured data point (epoch 11 of Barbary et al. 2008). We note that for this redshift, the lack of $\mathrm{H}$ or $\mathrm{He}$ features in the spectrum is an issue (Section 2). We also note that dust formation may play a role in the decline from the peak (Section 3.3).

\subsection{Special Case 2: $z=0.57$}

We now follow the same argument as before for the case of a redshift of 0.57. The results of this fit are shown in the bottom panel of Figure 2. The best fit gives an estimate of $M_{\mathrm{Ni}}=4.83 M_{\odot}$ and $M_{\mathrm{ej}}=19.68\left(\frac{\kappa}{0.1 \mathrm{~cm}^{-2} \mathrm{~g}^{-1}}\right)^{-1}\left(\frac{v_{\mathrm{ph}}}{10,000 \mathrm{~km} \mathrm{~s}^{-1}}\right)$ $M_{\odot}$. Note that the ejecta mass is less in this case than for $z=0.143$ because the dilation reduces the width of the light curve in the rest frame. Again the ejecta mass is rather large, but scales with the uncertain opacity and velocity. The nickel mass in this case is higher, as expected, but within the range of values predicted for other luminous $\mathrm{SNe}$ based on radioactive diffusion models (Quimby et al. 2007b; Smith et al. 2007). The best-fitting gamma-ray leakage parameter in this case yields a gamma-ray opacity of $\kappa_{\gamma}=0.03 \mathrm{~cm}^{2} \mathrm{~g}^{-1}$ which is in agreement with the generally assumed value of $0.03 \mathrm{~cm}^{2} \mathrm{~g}^{-1}$. Gamma-ray leakage has no substantial effect on the light curve.

The total ejecta mass in this case implies a kinetic energy equal to $E_{\mathrm{KE}}=1.2 \times 10^{52}\left(\frac{\kappa}{0.1 \mathrm{~cm}^{-2} \mathrm{~g}^{-1}}\right)^{-1}\left(\frac{v_{\mathrm{ph}}}{10,000 \mathrm{~km} \mathrm{~s}^{-1}}\right)^{3} \mathrm{erg}$, with a total radiated energy of $E_{\mathrm{rad}}=1.4 \times 10^{51} \mathrm{erg}$. While the 
radiated energy is somewhat large for a normal $\mathrm{SN}$, the kinetic energy is again quite large and comparable to that estimated for $z=0.143$. For the fiducial parameters in this case, the implication is again that much of the initial shock energy must have been lost to adiabatic expansion. If the opacity were larger than adopted here for illustration and the photospheric velocity somewhat smaller, as implied by the recombination models, the estimated kinetic energy could be more representative of normal $\mathrm{SNe}$, and the estimated radiated energy could be comparable to the estimated kinetic energy, in which case substantial adiabatic losses would not be implied. This would imply a large initial radius and thus perhaps a dense circumstellar medium as will be discussed below.

As for the lower-redshift case, the basic radioactive diffusion model does not produce the steep post-maximum decline. The dashed line in the bottom panel of Figure 2 shows a model with recombination at $10,000 \mathrm{~K}$ and $\kappa=0.2 \mathrm{~cm}^{2} \mathrm{~g}^{-1}$ which is appropriate for an $\mathrm{H}$-poor envelope (see Table 2). Dust formation might also play a role (Section 3.3). We expect no evidence for $\mathrm{H}$ to be observed at this redshift (Section 2).

\section{CSM INTERACTION}

Now we investigate the possibility of the contribution of CSM interaction producing optical/NIR emission in the observed light curve of SCP06F6. There are two versions of this circumstance. In the model of Smith \& McCray (2007), the CSM shell is optically thick, and the light curve is controlled by diffusion. Alternatively, the CSM could be optically thin enough to radiate the shock collision energy "instantaneously," but optically thick enough to convert the shock energy to optical radiation.

In the case where the energy release is dominated by diffusion, the energy to power the light curve arises in the putative collision of the SN ejecta with the extended dense CSM. There is no need for any radioactive input to power the light curve near maximum light, although some contribution from that source cannot be ruled out. This class of model gives no simple way to estimate the maximum luminosity in terms of physical input parameters. Those input parameters would be the initial radius and kinetic energy of the underlying $\mathrm{SN}$, and the initial radius and density distribution of the CSM. An extreme version of this class of model is obtained with the assumption that the initial radius of the circumstellar shell was not far from the observed radius of the photosphere at maximum light so that adiabatic losses within the shell are assumed to be minimal. In this case, the post-shock thermal energy content of the CSM envelope should be roughly comparable to the radiated energy and to the kinetic energy, and both should be roughly comparable to the initial SN shock energy, the kinetic energy of the underlying SN (within factors of 2). These aspects mean that, except for this "extreme" version, there is no simple way to determine a physical parameter analogous to the initial nickel mass in the radioactive diffusion model derived from the light-curve peak. On the other hand, this model still assumes that the luminous output derives from the diffusion of thermal energy from within the optically thick ejecta. In this case, the mass of the ejecta that determines the width of the light curve is roughly the sum of the underlying SN ejecta and the mass of the shocked CSM matter (Smith \& McCray 2007), and the tools presented in Section 3 still allow a determination of this effective mass as a function of redshift.

If the optical depth is small enough that the diffusion time for the release of post-shock energy is short, then the luminosity released as the CSM is shocked is given by (Ofek et al. 2007; Gezari et al. 2009; Smith et al. 2008)

$$
L \sim 2 \pi \rho_{\mathrm{CSM}} R^{2} v_{\mathrm{sh}}^{3}
$$

where $\rho_{\mathrm{CSM}}$ is the density of the CSM and $v_{\mathrm{sh}}$ is the velocity of the shock. Note that in this case, the luminosity is presumed to reflect the local density, and hence the shape of the light curve is given by the density distribution. The light curve can be reproduced by a suitable, although entirely ad hoc, assumption of a density profile. We also note that while some information on the mass ejected into the CSM can be estimated, this model provides no useful separate information on the ejecta mass analogous to the constraint of the rise time to maximum. Thus there is also no independent constraint on the associated kinetic energy.

For the CSM diffusion models, the width of the light curve yields an estimate of the diffusion time and hence the ejecta mass from Equation (4). An estimate of the radius of the shell from kinematics or emissivity (Figure 5) then yields an estimate of the mean density, $\bar{\rho}$. For the $z=0.143$ case, the radius at maximum light is estimated from the kinematics to be $R_{k, 0.143}=v_{\mathrm{ph}} t_{\max } /(1+z)=7.5 \times 10^{15} \mathrm{~cm}$ for a photospheric velocity of $10,000 \mathrm{~km} \mathrm{~s}^{-1}$. Assuming a temperature of $5000 \mathrm{~K}$ in the rest frame of the SN (Gaensicke et al. 2008), the blackbody radius at maximum light is $R_{\mathrm{bb}, 0.143}=1.8 \times 10^{15} \mathrm{~cm}$. The rather large discrepancy in these two methods for estimating the radius near maximum light suggests a basic inconsistency in the diffusion models for this smaller redshift. Following the same method for the $z=0.57$ case, we find that the blackbody radius is $R_{\mathrm{bb}, 0.57}=6.2 \times 10^{15} \mathrm{~cm}$ while the kinematic radius at maximum light is $R_{k, 0.57}=5.5 \times 10^{15} \mathrm{~cm}$. This represents generally good agreement. If the true blackbody radius at maximum light were much higher, this model might be strained.

The significantly low fraction of radiation energy compared to the kinetic energy for the model at $z=0.143, E_{\mathrm{th}} / E_{\mathrm{kin}} \sim 0.005$ (Section 4.3), implies that there must have been substantial adiabatic losses for this CSM diffusion model to be viable, unless the opacity is substantially larger than the fiducial value of $\kappa=0.1 \mathrm{~cm}^{2} \mathrm{~g}^{-1}$ (see Figure 4) and the photospheric velocity substantially lower. For the case at $z=0.57$, the radiated energy is again estimated to be small compared to the kinetic energy, but rather modest changes in the fiducial parameters (larger opacity, smaller velocity) could make the radiated energy comparable to the kinetic energy. In this case, the light curve could be accounted for by the collision of an underlying SN with a large, dense, CSM shell. For this situation, with negligible adiabatic losses, the energy of the underlying $\mathrm{SN}$ would be about twice the radiated energy, or about $3 \times 10^{51} \mathrm{erg}$. The estimate of optical depth near maximum light, $\tau_{\max } \simeq 30$, from Equation (5) also applies to this diffusion model. This constant value of opacity is given as the horizontal dashed line in Figure 6. Because a CSM envelope is likely to be of relatively large opacity, the shock diffusion model may be appropriate to this redshift. The expected composition of the CSM shell is hydrogen so the lack for $\mathrm{H}$ features in the spectrum is a constraint at $z=0.143$ but not necessarily at $z=0.57$ (Section 2).

An estimate of the optical depth can also be made in the context of the model for which the optical depth is the modest and the shock energy is radiated "instantaneously." In this case, we can estimate the density with which the shock collides near 


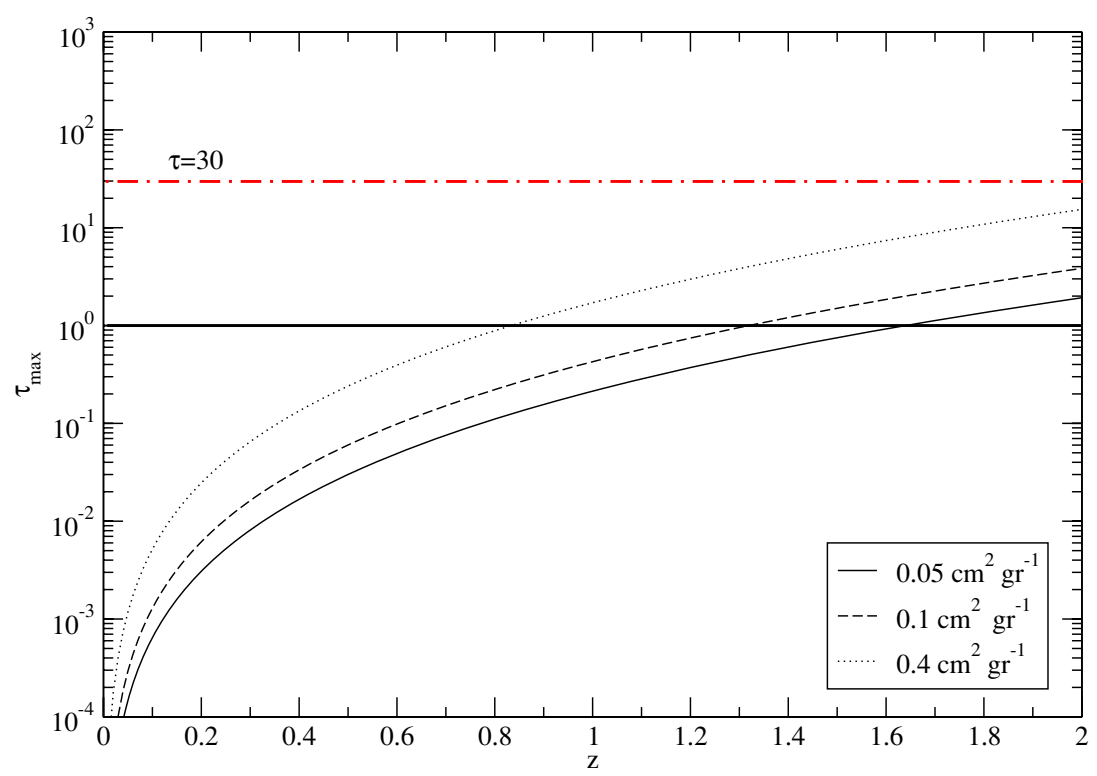

Figure 6. Dependence of the optical depth at maximum light on redshift for CSM models of SCPO6F6. The horizontal dot-dash line at $\tau=30$ represents the estimated optical depth $\left(\tau=c / v_{\mathrm{ph}}\right)$ for a diffusion shell model with radius given by the kinematic estimate (see the text). The solid horizontal line corresponds to $\tau=1$. The thin solid, dashed, and dotted lines are based on a model of moderate optical depth for which the luminosity is quickly radiated behind the shock. This model is valid only for $\tau \gtrsim 1$.

(A color version of this figure is available in the online journal.)

maximum light from Equation (5) as

$$
\rho\left(R_{\max }\right) \sim \frac{L_{\max }}{2 \pi R_{\max }^{2} v_{\mathrm{sh}}^{3}} .
$$

Taking the shock velocity to be constant and equal to the photospheric expansion velocity and hence $R_{\max }=v_{\mathrm{ph}} t_{\max }$ yields

$$
\tau \sim \frac{\kappa L_{\max }}{2 \pi v_{\mathrm{ph}}^{4} t_{\max }}
$$

with $t_{\max }=t_{m, \mathrm{obs}} /(1+z)$. Note that the optical depth for this model is very sensitive to the assumed shock velocity. Adopting a characteristic shock velocity of $10,000 \mathrm{~km} \mathrm{~s}^{-1}$, we can estimate $\tau$ for a given opacity. In Figure 6, we also show how the optical depth at maximum light estimated in this way scales with redshift. Note that the optical depth estimated based on this model increases rapidly with redshift. For optical depths less than unity, the luminosity is expected to be emitted at high-energy, non-optical wavelengths. For optical depths much greater that unity, the diffusion time, not shock propagation, will control the emission timescale. From Figure 6, the range of validity of this model for the fiducial parameters is $z \geqslant 0.9$. For the $z=0.57$ case, this model could be made self-consistent for higher opacity or smaller shock velocities. The lack of evidence for $\mathrm{H}$ in the spectrum again constrains this class of models, especially at lower redshifts.

We can also constrain the mass-loss rate associated with the CSM. For circumstances in which the CSM is optically thick and diffusion controls the light curve, we can use the estimates of the CSM mass and the radius of the configuration to make an estimate of the effective mass-loss rate. The mass-loss rate can be estimated as

$$
\dot{M}=\frac{M_{\mathrm{csm}}}{t_{\max } v_{\mathrm{ph}} / v_{w}},
$$

where $v_{w}$ is the velocity of the "wind" that led to the formation of the CSM. For a red-giant-type wind, this velocity might be
$10 \mathrm{~km} \mathrm{~s}^{-1}$. For an Luminous Blue Variable (LBV)-type massloss event, a typical velocity might be $\sim 100 \mathrm{~km} \mathrm{~s}^{-1}$ (Smith et al. 2004; Smith 2006). If we assume that the diffusion time is dominated by the mass ejected into the CSM prior to explosion, and hence that the "ejected" mass in Figure 2 is a measure of the CSM mass, then we can estimate the effective mass-loss rate. For $z=0.143$, we get $0.15 M_{\odot} \mathrm{yr}^{-1}$ and $1.5 M_{\odot} \mathrm{yr}^{-1}$ for $v_{w}$ equal to 10 and $100 \mathrm{~km} \mathrm{~s}^{-1}$, respectively. For $z=0.57$, we get $0.11 M_{\odot} \mathrm{yr}^{-1}$ and $1.11 M_{\odot} \mathrm{yr}^{-1}$. These values were all based on our fiducial opacity and so scale as $\left(\frac{\kappa}{0.1 \mathrm{~cm}^{-2} \mathrm{~g}^{-1}}\right)^{-1}$. For a Wolf-Rayet progenitor the wind velocity could be up to 1000 $\mathrm{km} \mathrm{s}^{-1}$ implying an even larger mass-loss rate. All these values are large, implying a more LBV-like process if this diffusion model is pertinent.

We can also estimate the mass-loss rate implied in the model where the luminosity arises by shock interaction in a CSM of modest optical depth. The total envelope mass within a radius $R=v_{\mathrm{sh}} t_{\max }$ is, with Equation (7),

$$
M_{\mathrm{csm}}=\frac{4}{3} \pi R^{3} \rho \simeq \frac{2}{3} \frac{L_{\mathrm{max}} t_{\mathrm{max}}}{v_{\mathrm{sh}}^{2}} .
$$

With Equation (9), we can then write

$$
\dot{M}=\frac{2}{3} \frac{L_{\max } v_{w}}{v_{\mathrm{sh}}^{3}} \sim 10^{-4} M_{\odot} \mathrm{yr}^{-1} \frac{L_{\mathrm{max}, 42} v_{w, 100}}{v_{\mathrm{sh}, 10,000}^{3}},
$$

where $L_{\max , 42}$ is the peak luminosity in $10^{42} \mathrm{erg} \mathrm{s}^{-1}, v_{w, 100}$ is the wind velocity in units of $100 \mathrm{~km} \mathrm{~s}^{-1}$, and $v_{\mathrm{sh}, 10,000}$ is the shock speed in units of $10,000 \mathrm{~km} \mathrm{~s}^{-1}$. For a redshift of 0.57 , the corresponding mass-loss rate is about $3 \times 10^{-3} v_{w, 100} v_{\text {sh } 10,000}^{-3}$ $M_{\odot} \mathrm{yr}^{-1}$. This mass-loss rate could be representative of a WolfRayet star progenitor, but then the wind velocity should be higher to correspond to observed Wolf-Rayet stars. Another difficulty is that this mass distribution cannot be a standard $\rho \propto r^{-2}$ wind as roughly expected for a Wolf-Rayet star. Rather, the density profile must be carefully "designed" to reproduce 
the shape of the light curve. This conceptual problem mitigates against this model on general grounds.

Gaensicke et al. (2008) reported a very high $0.5-10 \mathrm{keV} X$-ray flux near an optical maximum of about $10^{-13} \mathrm{erg} \mathrm{s}^{-1} \mathrm{~cm}^{-2}$. This is nearly four times the bolometric flux. It is possible that the reported detection should instead be treated as an upper limit (D. Pooley 2009, private communication). The X-ray flux could arise from shocked optically thin CSM. In this case, Equations (5) and (7) pertain, and if we take the reported X-ray flux at the face value, we estimate particle densities $6.4 \times 10^{6}$ $\mathrm{cm}^{-3}$ and $2.6 \times 10^{8} \mathrm{~cm}^{-3}$ for redshifts of 0.143 and 0.57 , respectively, for a shock velocity of $10,000 \mathrm{~km} \mathrm{~s}^{-1}$. This density is $100-10,000$ times smaller than the average density $\bar{\rho}$ of a dense optically thick shell for the two redshift cases. This result implies that the observed X-ray flux, if real, might possibly arise in the outer, lower-density portion of the diffusive shell that produces the optical display. For a CSM of modest optical depth, it is difficult to see how a self-consistent model based on shock interaction could simultaneously explain the optical and the X-ray luminosity. A possibility is that the medium is clumpy with dense clumps providing the optical emission and inter-clump regions providing X-rays.

\section{DISCUSSION AND SUMMARY}

We discussed the applicability of some widely used SN lightcurve models in reproducing the observed light curve of the luminous peculiar transient SCP06F6 discovered by Barbary et al. (2008). The parameters estimated based on various models are summarized in Table 1. The observed light curve can be approximated by a smooth simple diffusion model, but the rapid decline from maximum is not consistent with the simple models. Fits to a radioactive decay diffusion model provide estimates for the ejecta mass, the nickel mass, and the gamma-ray opacity versus redshift depending on the key, but uncertain parameters, especially the mean optical opacity and photospheric velocity. We also note that while the data invite the interpretation of a smooth rise and decline of the light curve, the photometric data are sparse. The possibility of a precursor peak, such as displayed by SN1987A or SN2005bf, cannot be ruled out. The existence of such a feature would modify any interpretation of the data.

We considered two specific choices of the redshift, $z=$ 0.143 based on a suggestion by Gaensicke et al. (2008) and $z=0.57$, based on a crude fit to $\mathrm{Ca} H \& \mathrm{~K}$ and iron-peak absorptions in various SNe. Substantially higher redshifts, greater than $\sim 1.1$, do not lead to self-consistent results in the context of the radioactive decay diffusion model: the nickel mass becomes too large compared to the ejecta mass and the radiated energy exceeds the kinetic energy. For these two specific choices of redshift, we found nickel masses of 0.28 and $4.83 M_{\odot}$, respectively, and ejecta masses of $\sim 37$ and $\sim 20 M_{\odot}$, respectively, for an adopted mean opacity of $0.1 \mathrm{~cm}^{2} \mathrm{~g}^{-1}$ and a photospheric velocity of $10,000 \mathrm{~km} \mathrm{~s}^{-1}$. The estimated ejecta mass scales inversely with the opacity and directly with the velocity. The results are roughly commensurate with normal core-collapse $\mathrm{SNe}$ at lower redshift, but at the larger redshift, the nickel mass would require a different situation, perhaps similar to that invoked for some models of SN 2006gy based on pair-formation $\mathrm{SNe}$ (Smith et al. 2007). For both redshifts, the implied kinetic energy is very high, $\sim 10^{52} \mathrm{erg} \mathrm{s}^{-1}$ for the fiducial parameters, but would be less for smaller ejecta velocities. In either case, our simple diffusion models do not account for the rapid post-maximum decline. Some other factor would need to be invoked such as a change in opacity or dust formation. We show that recombination of $\mathrm{H}$, maybe $\mathrm{He}$, might plausibly account for a decline in opacity and hence in the light curve.

We also consider models in which the optical luminosity is provided by collision of the SN with a dense CSM shell in the spirit of Smith \& McCray (2007) or models in which the shock energy is thermalized, but rapidly radiated away (Ofek et al. 2007; Gezari et al. 2009; Smith et al. 2008). In general, the models with dense, optically thick extended shells behave in a manner similar to that of the radioactive decay diffusion models, except that the energy to heat the CSM envelope is presumed to derive from the kinetic energy of the underlying explosion, rather than radioactive decay. These models applied at low redshift give the radiated energy substantially less than the kinetic energy, implying that to be self-consistent, the CSM envelope must have undergone substantial adiabatic expansion after being shocked. The same is true for the model applied at larger redshifts, but reasonable choices of the parameters would yield a model for which the shell had expanded little by maximum light. For such a choice of parameters, the energy of the underlying explosion could be more modest. These models demand substantial mass in the CSM and hence effective massloss rates that are reminiscent of LBV mass-loss episodes. Models in which the CSM is only modestly optically thick so the energy can be thermalized but also radiated rapidly could be applicable at higher redshifts. Such models might be consistent with mass-loss rates reminiscent of Wolf-Rayet stars, but to match the shape of the light curve, the density profile would need to be carefully contrived, an unlikely happenstance.

The spectrum of SCP06F6 does not resemble any other transient spectrum that has been obtained so far. The four broad absorption features can be reasonably well fit by the molecular $\mathrm{C}_{2}$ Swan bands of a carbon-rich star of temperature 3000$10,000 \mathrm{~K}$ (Gaensicke et al. 2008). Swan bands are also observed in white dwarfs at substantially higher temperatures, but only because of the very high gravity that would not pertain to SN ejecta. Pending the calculation of a realistic SN atmosphere model showing that $\mathrm{C}_{2}$ can form and survive, we find this hypothesis intriguing, but unlikely, at least around maximum light when the ejecta is supposed to have $T_{\text {eff }} \simeq 5000 \mathrm{~K}$. On the other hand, at later phases, when the ejecta has cooled, the formation of $\mathrm{C}_{2}$ and other molecules, even dust grains, is indeed a possibility although this would imply the presence of $\mathrm{CN}$ ( $\lambda 7900$ rest frame) which we do not detect in the co-added VLT, Subaru, and Keck spectra of SCP06F6.

We propose that a model of SCP06F6 worth further consideration is one in which the event is at a redshift of $z \sim$ 0.57 for which the blue absorption features are $\mathrm{Ca} H \& \mathrm{~K}$ and iron-peak absorption features as qualitatively seen in the UV of some nearby SNe. At this redshift, the light curve can be reproduced semi-quantitatively with a diffusion model based either on radioactive decay requiring several solar masses of nickel or collision of a moderately energetic SN with a dense CSM envelope.

The characteristics of SCP06F6 that are deduced from its light curve at $z=0.57$ indicate that its luminosity is one order of magnitude less than that of the exceptionally luminous 2005ap, $2006 \mathrm{gy}, 2008 \mathrm{es}$, and 2005tf. This means that if SCP06F6 is a SN fitting the characteristics presented here, defining a new class of objects is not necessary to account for it. It fits well within the rubric of these other bright events. 
We are grateful to Kyle Barbary, Robert Quimby, Andy Howell, Nick Suntzeff, Alicia Soderberg, Dave Pooley, Milos Milosavljevic, and Volker Bromm for useful comments and suggestions and to the anonymous referee whose insightful queries substantially improved the manuscript. This research is supported in part by NSF Grant AST-0707669 and by the Texas Advanced Research Program grant ASTROARP-0094. E. Chatzopoulos would like to thank Propondis foundation of Piraeus, Greece for its support of his studies. J. Vinko received support from the Hungarian OTKA Grant K76816.

\section{REFERENCES}

Agnoletto, I., et al. 2008, arXiv:0810.0635

Arnett, D. 2008, in AIP Conf. Ser. 1053, Observational Evidence for Black

Holes in the Universe, ed. S. K. Chakrabarti \& A. S. Majumdar (Melville, NY: AIP), 237

Arnett, W. D. 1982, ApJ, 253, 785

Arnett, W. D., \& Fu, A. 1989, ApJ, 340, 396

Barbary, K., et al. 2008, arXiv:0809.1648

Barkat, Z., Rakavy, G., \& Sack, N. 1967, Phys. Rev. Lett., 18, 379

Bufano, F., et al. 2009, arXiv:0906.0367

Colgate, S. A., Petschek, A. G., \& Kriese, J. T. 1980, ApJ, 237, L81

Dolphin, A. E., et al. 2001, ApJ, 550, 554

Downes, R. A., et al. 2004, AJ, 127, 2838

Draine, B. T. 2003, ApJ, 598, 1017

Ercolano, B., Barlow, M. J., \& Sugerman, B. E. K. 2007, MNRAS, 375, 753
Gaensicke, B. T., Levan, A. J., Marsh, T. R., \& Wheatley, P. J. 2008 arXiv: 0809.2562

Gezari, S., et al. 2009, ApJ, 690, 1313

Gilliland, R. L., Nugent, P. E., \& Phillips, M. M. 1999, ApJ, 521, 30

Harkness, R. P., \& Wheeler, J. C. 1987, BAAS, 19, 740

Kirshner, R. P., et al. 1993, ApJ, 415, 589

Kotak, R., Meikle, P., van Dyk, S. D., Höflich, P. A., \& Mattila, S. 2005, ApJ, $628, \mathrm{~L} 123$

Kotak, R., et al. 2009, arXiv:0904.3737

Miller, A. A., et al. 2009, ApJ, 690, 1303

Ofek, E. O., et al. 2007, ApJ, 659, L13

Quimby, R. 2006, Central Bureau Electronic Telegrams, 644, 1

Quimby, R. M., Aldering, G., Wheeler, J. C., Höflich, P., Akerlof, C. W., \& Rykoff, E. S. 2007a, ApJ, 668, L99

Quimby, R., Castro, F., \& Mondol, P. 2007b, IAU Circ., 8790, 2

Quimby, R., Castro, F., Mondol, P., Caldwell, J., \& Terrazas, E. 2007c, Central Bureau Electronic Telegrams, 793,

Rakavy, G., \& Shaviv, G. 1967, ApJ, 148, 803

Smith, N. 2006, ApJ, 644, 1151

Smith, N., Chornock, R., Li, W., Ganeshalingam, M., Silverman, J. M., Foley, R. J., Filippenko, A. V., \& Barth, A. J. 2008, ApJ, 686, 467

Smith, N., Chornock, R., Silverman, J. M., Filippenko, A. V., \& Foley, R. J. 2009, arXiv:0906.2200

Smith, N., \& McCray, R. 2007, ApJ, 671, L17

Smith, N., Vink, J. S., \& de Koter, A. 2004, ApJ, 615, 475

Smith, N., et al. 2007, ApJ, 666, 1116

Soderberg, A. M., et al. 2008, Nature, 453, 469

Soker, N., Frankowski, A., \& Kashi, A. 2008, arXiv:0812.1402

Sutherland, P. G., \& Wheeler, J. C. 1984, ApJ, 280, 282

Tominaga, N., et al. 2005, ApJ, 633, L97

Valenti, S., et al. 2008, MNRAS, 383, 1485

Woosley, S. E., Blinnikov, S., \& Heger, A. 2007, Nature, 450, 390

Yuan, F., et al. 2008, Central Bureau Electronic Telegrams, 1462, 1 